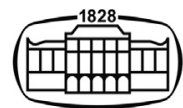

AKADÉMIAI KIADÓ

\title{
Knowledge, awareness, and usage of probiotics among Hungarian adults: An explorative survey
}

\author{
L. HAVELDA ${ }^{1 *}$, Z. BENCZ $^{2}$ and M. VERESNÉ BÁLINT ${ }^{1}$
}

\section{Developments in Health Sciences}

$3(2020) 3,53-57$

DOI:

$10.1556 / 2066.2020 .00010$

(c) 2020 The Author(s)

\section{ORIGINAL RESEARCH} PAPER

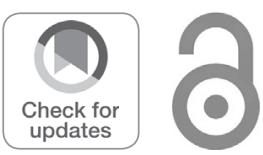

*Corresponding author. Department of Dietetics and Nutrition Sciences, Faculty of Health Sciences, Semmelweis University, Vas utca 17, Budapest $\mathrm{H}-1088$, Hungary. E-mail: haveldaluca97@gmail.com

\author{
${ }^{1}$ Department of Dietetics and Nutrition Sciences, Faculty of Health Sciences, Semmelweis University, \\ Budapest, Hungary \\ ${ }^{2}$ Vitaminkosár Ltd., Budapest, Hungary
}

Received: June 10, 2020 • Revised manuscript received: August 7, 2020 • Accepted: September 24, 2020

Published online: November 19, 2020

\begin{abstract}
Purpose: The aim of this study was to evaluate the use of probiotic products in Hungary, to explore consumers' knowledge of probiotics, and to gain information about the main characteristics of current usage. Materials and Methods: The research used a self-developed explorative questionnaire to obtain the necessary information. The data collected was further analysed with IBM SPSS 25.0 and Microsoft Office Excel. Results: Nearly three-quarters of respondents (73.9\%) have used probiotics in their life. Those who have already used these products, mostly heard about them from doctors (62.8\%) and friends/relatives $(40.5 \%)$, while among non-users the main sources of information were web pages (46.8\%) and commercials (45.2\%). Conclusions: We found several differences between users and nonusers in terms of probiotics awareness. Consequently, healthcare providers may play an important role in the process of careful product choice and proper usage.
\end{abstract}

\section{KEYWORDS}

probiotics, survey method, dietary supplements, consumer preferences

\section{INTRODUCTION}

To date, the commonly accepted definition of probiotics is the one that was created by the Food and Agriculture Organization of the United Nations and the World Health Organization. According to this definition probiotics are "live microorganisms that, when administered in adequate amounts, confer a health benefit on host" [1].

The number of publications related to probiotics have increased excessively in the last few decades. These research studies are testing the possible therapeutic and preventive effects of these products in several fields, but the results of these experiments and surveys are not easily comparable because of the use of different methods, probiotic strains and means of administration [2,3]. Besides this extensive scientific interest, the probiotic market is also expanding continuously, showing the increasing popularity of probiotic products among non-professional consumers as well. Most of these goods are non-prescription products and are placed on the market mainly as food or dietary supplements [4]. Consequently, neither the right choice of the products, nor their appropriate usage is controlled by healthcare professionals in all cases. Since the effect of probiotics is strain specific and greatly dependent on the dose as well, not all the available products are equally effective for a specific health problem [3]. Therefore, it should be important that the consumers have the correct information about the appropriate choice of products, label interpretation and adequate usage, particularly because the regulation of these categories is much less strict than if they were pharmaceutical products.

Despite the widespread use of probiotics, only a few articles have been published so far about the current pattern of probiotic usage among non-professionals. To summarise the former studies, we can say that mostly they were region-based, which means that the data collection usually took place only in one town or country and thus the sample sizes were 
limited. Despite the hardly comparable results, which are caused by the different sampling and survey methods as well as the demographic and cultural differences, there are some tendencies and characteristics which can be drawn from the previous researches.

The proportion of probiotic users in the population is increasing continuously. In 2011 Schultz et al. found that only one-quarter $(25.4 \%)$ of the respondents had tried probiotics in the past, while this proportion was higher in several following research studies, for example, in a study from 2015, where more than half of the participants (53\%) used a product earlier $[5,6]$. According to these prior studies, not only the rate of the usage increased over time, but more and more people tend to believe that they are familiar with the term "probiotic" [7, 8]. Some studies have shown that a relationship between the assumed knowledge and choosing the proper definition is not always present. Although $43 \%$ of the respondents stated that they are aware of the meaning of "probiotics" in the research of Betz et al. in 2015 , only half of them $(20 \%)$ were able to choose the correct definition [5]. The younger population and those who have higher education level seemed to have more knowledge about probiotics and their proportion was higher among probiotic users as well $[6,9]$. The respondents of these studies usually used probiotics to maintain their gastrointestinal health and to cure or prevent the side effects of an antibiotic treatment $[6,7]$. The participants have heard about probiotics and obtained their related knowledge usually from different media sources, but family members, friends, and physicians were also common information providers [5-7]. Most of the non-users would be willing to try out these goods if their physician advised them [7]. The majority of the respondents consumed them as food products (yoghurt, fermented milk), while the tablet and capsule forms were not that popular among them $[6,9,10]$.

\section{MATERIALS AND METHODS}

\section{The questionnaire}

This research used a self-developed explorative survey to obtain the necessary information. The questionnaire included demographic questions and questions regarding respondents' knowledge on probiotics and their product usage. It consisted of closed and open-ended questions and in some cases 1-5 scales were available for them, where they were informed about the meaning of the two terminal values (1 and 5) and therefore they were able to choose the value most representative of their opinion. During the survey nonusers and users needed to answer different questions.

\section{Data collection}

The data collection was carried out in two channels: The respondents could fill out the questionnaire online and the participants of an Elderly Academy had the opportunity to answer the questionnaire in a printed form, although most of them preferred the online option. The survey was shared online on Facebook and some mailing lists. Eventually 306 questionnaires were completed and returned between the middle of September 2019 and the end of October 2019. The questionnaires were completed anonymously and only adults (18 years of age or older) were enrolled.

\section{Data analyses}

We used the IBM SPSS Statistics for Windows, Version 25.0 (IBM Corp. Released 2017, Armonk, NY: IBM Corp.) and Microsoft Office Excel 2010 to process the collected data. Descriptive statistical methods were used to determine the frequency of the answers and we also used crosstabs analysis to examine the relation between different subgroups, such as age, education level, and the rate of probiotic use among them. We worked with $95 \%$ confidence level during the statistical analysis.

\section{RESULTS}

\section{Participant characteristics}

306 questionnaires were completed and returned. The majority of the respondents were female $(n=238,77.5 \%)$ and were younger than 30 years old ( $n=194,63.4 \%)$. Typically, their BMI (Body Mass Index) was in the normal range $(n=$ $189,61.8 \%)$ and most commonly they lived in the capital city $(n=131,42.8 \%)$ or in other towns $(n=118,38.6 \%)$. Most of them had completed their high school education ( $n=289,94.4 \%)$ and nearly half of them also had a university degree $(n=135,44.1 \%)$.

\section{The prevalence of probiotic use among the respondents}

The results showed that nearly three-quarters of participants ( $n=226,73.9 \%$ ) had used some kind of probiotic product in their life, one-fifth of them $(n=62,20.3 \%)$ knew about them but never used them and only $5.9 \%(n=18)$ had never even heard the term "probiotic". There was no statistically significant relation between the probiotic product usage and education level or age group.

\section{Probiotic knowledge among the respondents}

Most of the respondents rated their knowledge as good or very good (scores 4 and 5 on a $1-5$ scale) ( $n=184,63.9 \%)$. In the following question they needed to decide what probiotic products contain. Among the answers the definitions of not only probiotics but also of prebiotics, synthetic active agents, herbal active agents. and vitamins/minerals were listed. From these options the respondents were able to choose more than one. The most popular answer, according to the marking frequency was the definition of probiotics ( $n=225,78.1 \%$ ), which means that more than three-quarters of the participants knew what a probiotic product contains, but it is important to note that several respondents chose the definition of prebiotics as well $(n=195,67.7 \%)$ (Fig. 1). 


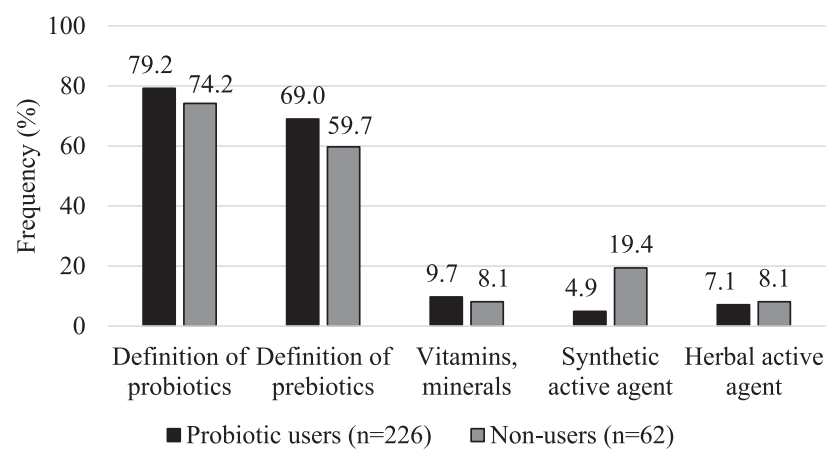

Fig. 1. Frequency of categories on "What do probiotic products contain?" by the usage of probiotic products $(n=288)$

\section{The sources of information on probiotics among the respondents}

The majority of respondents heard about probiotics from physicians $(n=145,52.3 \%)$, family members/friends $(n=101,36.5 \%)$ or saw them in commercials $(n=95$, $34.3 \%)$. We detected several differences between non-users and users and found statistically significant relations. Those who had used a product earlier had heard about them from physicians $(P<0.001)$, pharmacists $(P<0.001)$, and family members/friends $(P=0.011)$ in greater proportions. In contrast to this, non-users heard about them in greater proportion from the Internet $(P=0.009)$ and commercials $(P=0.049)$. There was no statistically significant relation found in the case of healthcare professionals (dietitians, nurses, etc.) as sources of information (Fig. 2).

\section{The characteristics of probiotic use}

The most common reasons of usage among the participants were to mitigate the side effects of previous antibiotic treatment $(n=198,87.6 \%)$ and the symptoms of diarrhoea $(n=78,24.5 \%)$. More than half of those who have never tried these products would be willing to try them out $(n=50$,

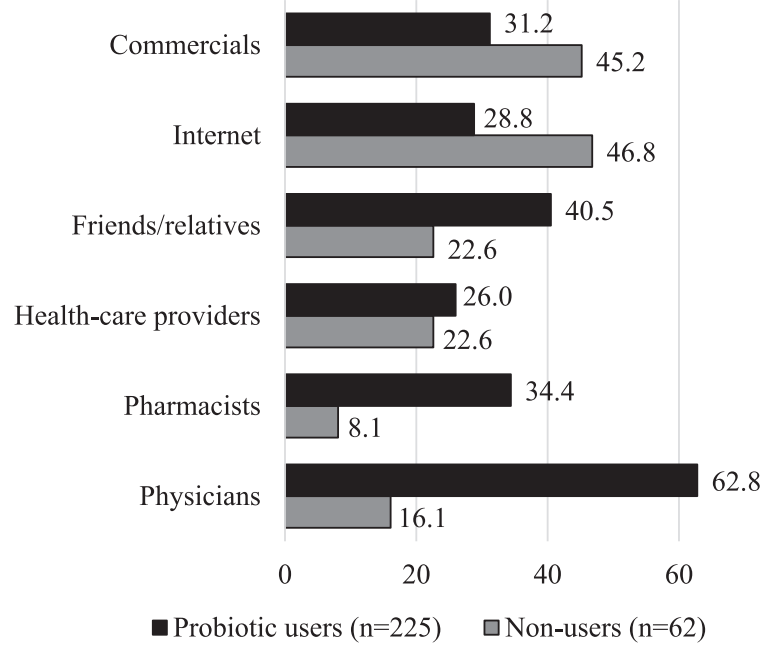

Fig. 2. Frequency of categories on "From whom did you hear about probiotics?" by the usage of probiotic products $(n=287)$
62.5\%) mainly to cure the side effects of antibiotic treatment ( $n=49,62.3 \%)$, the symptoms of diarrhoea $(n=27,34.6 \%)$ and to strengthen the immune system $(n=28,35.9 \%)$.

\section{Consumer preferences}

Most of the respondents would consume probiotics as food products $(n=215,70.5 \%)$, but the capsule form $(n=152$, $49.8 \%)$ and liquid form $(n=106,34.8 \%)$ were also popular options. The majority of them would buy these goods in pharmacies $(n=190,86.4 \%)$ and with their questions related to probiotics they would turn mostly to their physicians ( $n=244,79.9 \%)$, pharmacists $(n=215,70.3 \%)$, and other healthcare professionals, such as dietitians or nurses ( $n=151,49.3 \%$ ), but the Internet was also chosen by $27.8 \%$ of the respondents $(n=85)$.

\section{DISCUSSION}

The purpose of this study was to evaluate the use of probiotic products in Hungary and to gain information about the main characteristics of consumers' current usage and knowledge on probiotics.

According to our results, almost three-quarters $(n=226$, $73.9 \%$ ) of respondents used probiotics in the past, which is a much higher rate than what can be found in the literature. In a New Zealand cohort study, in 2011 this rate was only $25.4 \%$, while later research studies reported higher consumer proportions, which shows a continuous increase in the usage of these goods $[5,6]$.

In contrast to most of the previous studies, we did not find relations between demographic variables and probiotic use $[5,6]$. Neither the younger population, nor those who had higher education level seemed to use probiotics in a significant larger proportion than the other respondents. The difference can be explained by one of our sampling methods, since active university students were overrepresented in our sample while they were categorised as "possessing high school degree".

Our results showed that the majority of the respondents believed that they are aware of what the term "probiotics" means and most of them were able to choose the correct definition as well. Therefore, it seems that they know what "probiotic" means, which was not usual in prior studies. For instance, in 2015, in Betz et al. research, only $20 \%$ of the respondents chose the proper definition, although $43 \%$ stated that they are aware what the term "probiotic" means [5]. Our surprisingly high rate of correctly choosing the definition may have been caused by the nature of this question, since it was a multiple choice question. Besides the popularity of the definition of probiotics among the answers, a similar proportion of the participants chose the definition of prebiotics for probiotics; therefore, we can assume that the concepts of these two phrases are not entirely clear for every respondent who falls into the group that marked the correct definition of probiotics and they may use these words interchangeably. 
The most common reasons of usage among the respondents were to mitigate the side effects of previous antibiotic treatment and the symptoms of diarrhoea. Although in the literature the most cited reason of probiotic use was also antibiotic treatment, other promoter factors were mentioned in a great number as well, such as oralhealth, the maintenance of gut flora, cardiovascular health, and immune health, which were chosen by the participants of our study as well, but not in a large proportion $[5-7,10]$. This difference may be explained with the relevant Hungarian commercials. These usually highlight the beneficial effects of probiotics in connection with antibiotic treatment.

More than half of those who have never used a product before would be willing to try out these goods, which is a similar proportion that can be found in the literature [8].

In line with prior studies the main sources of information on probiotics were physicians, friends/relatives, commercials, and the Internet, but we found several differences between probiotic users and non-users since the most popular sources of information were physicians and pharmacists among those who used a product earlier [5-7]. We can assume that these consumers used the proper product and in a correct way to manage their health problems, because professionals had a role in the process of product selection. In contrast, the non-users mostly obtained their information from the Internet and commercials. From these sources it cannot be stated that they are wholly reliable and valid, therefore, it is not guaranteed that these participants gained proper information about probiotics, their usage, and whether their use was justifiable in that particular health problem.

In the present study we found that the majority of respondents would turn to healthcare professionals (physicians, pharmacist, dietitians, nurses, etc.), which is similar to the findings of Chin-Lee et al. [7] and our findings also show the need for consumers to acquire adequate information about probiotics and the responsibility of professionals in this process.

Our quantitative study used a complex approach to obtain the necessary information, however, in our sample both women and the younger population were overrepresented. Another limitation of our study was the use of general questions, but with this approach the solid base for further investigations and more specific research could be established.

\section{CONCLUSIONS}

In summary, we found that the use of probiotics among the respondents was relatively high. Most of them are familiar with the term of probiotics, but information available for them may not always be entirely correct. Healthcare professionals may play an important part in the discussion on and the suggestion of dietary supplements such as probiotics and in providing proper information about their risks and benefits and about product usage to future consumers.

To make this process easier for both healthcare professionals (e.g., General Practitioners) and non- professionals, it would be beneficial to create an online upto-date database and professionally reliable websites. These platforms would make appropriate information accessible about the probiotic strains, their benefits and risks and even about those products which are currently on the probiotic market and are available for consumers. These online sources of information and training courses on probiotics for healthcare professionals would make the recommendation much faster, easier, and more precise. For non-professionals, informative flyers and presentations may be able to provide more scientifically controlled information.

Authors' contribution: LH conceptualised the study, reviewed the scientific literature, designed the questionnaire, collected the data, carried out the statistical analyses, and summarised the results. $\mathrm{ZB}$ conceptualised the study, designed the questionnaire, supervised the data collection, critically reviewed the manuscript, and approved the final manuscript. MVB conceptualised the study, designed the questionnaire, reached participants through the Elderly Academy, supervised the data collection, critically reviewed the manuscript, and approved the final manuscript.

Ethical approval: This study was conducted in accordance with the 2008 revision of the 1975 Declaration of Helsinki.

Conflict of interest/funding: The authors declare no conflicts of interest and no financial support was received for this study.

\section{REFERENCES}

1. Hill C, Guarner F, Reid G, Gibson GR, Merenstein DJ, Pot B, et al. The International Scientific Association for Probiotics and Prebiotics consensus statement on the scope and appropriate use of the term probiotic. Nat Rev Gastroenterol Hepatol 2014;11:506-14. https://doi.org/10.1038/nrgastro.2014.66.

2. Khalesi S, Bellissimo N, Vandelanotte C, Williams S, Stanley D, Irwin C. A review of probiotic supplementation in healthy adults: helpful or hype? Eur J Clin Nutr 2019;73:24-37. https://doi.org/10. 1038/s41430-018-0135-9.

3. McFarland LV. From yaks to yogurt: the history, development, and current use of probiotics. Clin Infect Dis 2015;60:S85-90. https:// doi.org/10.1093/cid/civ054.

4. de Simone C. The unregulated probiotic market. Clin Gastroenterol Hepatol 2019;17:809-17. https://doi.org/10.1016/j.cgh. 2018.01.018.

5. Betz M, Uzueta A, Rasmussen H, Gregoire M, Vanderwall C, Witowich G. Knowledge, use and perceptions of probiotics and prebiotics in hospitalised patients. Nurt Diet 2015;72:261-6. https:// doi.org/10.1111/1747-0080.12177.

6. Schultz M, Baranchi A, Thurston L, Yu YC, Wang L, Chen J, et al. Consumer demographics and expectations of probiotic therapy in New Zealand: results of a large telephone survey. N Z Med J 2011; 124:36-43. 
7. Chin-Lee B, Curry WJ, Fetterman J, Graybill MA, Karpa K. Patient experience and use of probiotics in community-based health care settings. Patient Prefer Adherence 2014;8:1513-20. https:/doi.org/ 10.2147/PPA.S72276.

8. Stanczak M, Heuberger R. Assessment of the knowledge and beliefs regarding probiotic use. Am J Health Educ 2009;40:207-11. https:// doi.org/10.1080/19325037.2009.10599095.
9. Kolady D, Kattelmann K, Vukovich C, Scaria J. Awareness and use of probiotics among the millennials in the United States: drivers and implications. FFHD 2018;8:505-18. https://doi.org/10.31989/ FFHD.V8I10.536.

10. Carvalho NB, da Costa TMT, Ferreira MAM, Simiqueli AA, Minim VPR. Consumer attitude regarding products containing probiotics. Cienc Rural 2014;44:1319-26. https://doi.org/10.1590/0103-8478cr20131178.

Open Access. This is an open-access article distributed under the terms of the Creative Commons Attribution 4.0 International License (https://creativecommons.org/ licenses/by/4.0/), which permits unrestricted use, distribution, and reproduction in any medium, provided the original author and source are credited, a link to the CC License is provided, and changes - if any - are indicated. (SID_1) 